\title{
Effect of enhanced recovery after surgery protocol on recovery after open hepatectomy: a randomized clinical trial
}

\author{
Wataru Nakanishi, Shigehito Miyagi, Kazuaki Tokodai, Atsushi Fujio, Kengo Sasaki, Yoshihiro Shono, \\ Michiaki Unno, Takashi Kamei
}

Department of Surgery, Tohoku University Graduate School of Medicine, Sendai, Japan

\begin{abstract}
Purpose: Enhanced recovery after surgery (ERAS) is beneficial to patients undergoing digestive surgery. However, its efficacy in patients undergoing open hepatectomy remains unclear.

Methods: Consecutive patients scheduled for open hepatectomy were randomly assigned to undergo either ERAS or conventional postoperative management. The primary endpoint was the amount of time that elapsed before patients were considered medically fit for discharge (MFD) and length of hospital stay (LOHS). Secondary endpoints included morbidity, mortality, the time to first flatus, defecation, first walk, and freedom from infusion. Perioperative serum nutritional markers, insulin resistance, respiratory quotient (RQ), and resting energy expenditure (REE) were also assessed.

Results: Between August 2014 and March 2017, 57 patients were randomized into 2 groups; ERAS group $(n=29)$ and conventional management $(n=28)$. The median MFD was not significantly different between the ERAS and conventional management groups (6.5 vs. 7 days; $P=0.381)$. Recovery from gastrointestinal paresis was significantly quicker in the ERAS group (1.8 vs. 2.4 days; $P=0.004$ ). There were no significant differences in serum markers, insulin resistance, $R Q$, and REE.

Conclusion: This trial did not demonstrate greater efficacy of the ERAS protocol following open hepatectomy in terms of the MFD and LOHS. However, the ERAS protocol was associated with better recovery from postoperative gastrointestinal paresis, suggesting that it is useful for patients undergoing open hepatectomy.

[Ann Surg Treat Res 2020;99(6):320-328]
\end{abstract}

Key Words: Enhanced recovery after surgery, Hepatectomy, Perioperative treatments, Randomized controlled trial

\section{INTRODUCTION}

Enhanced recovery after surgery (ERAS) [1] improved the time to recovery from surgical stress. Guidelines for perioperative care of patients undergoing digestive surgery were reported by the European Society for Clinical Nutrition and Metabolism in 2012, and are now accepted in more than 30 countries [2]. ERAS protocols aim to reduce perioperative surgical stress, maintain physical function, and promote postoperative rehabilitation.
Several studies have demonstrated that ERAS protocols can reduce the length of hospital stay (LOHS) for patients undergoing colorectal surgery [3,4].

Hepatectomy is commonly performed as a curative therapy mainly for the treatment of hepatocellular carcinoma (HCC) and colorectal metastases. The postoperative mortality rates after hepatectomy are low; however, morbidity rates remain high even at high volume centers [5]. These findings show the importance of improving perioperative care to reduce morbidity
Received May 14, 2020, Revised July 16, 2020, Accepted August 21, 2020 Corresponding Author: Wataru Nakanishi

Department of Surgery, Tohoku University Graduate School of Medicine, 1-1 Seiryo-machi, Aoba-ku, Sendai, Miyagi 980-0872, Japan

Tel: +81-22-717-7214, Fax: +81-22-717-7127

E-mail: wataru.nakanishi@gmail.com

ORCID: https://orcid.org/0000-0002-7034-9764
Copyright (c) 2020, the Korean Surgical Society

(c) Annals of Surgical Treatment and Research is an Open Access Journal. All articles are distributed under the terms of the Creative Commons Attribution NonCommercial License (http://creativecommons.org/licenses/by-nc/4.0/) which permits unrestricted non-commercial use, distribution, and reproduction in any medium, provided the original work is properly cited. 
rates among patients undergoing hepatectomy. ERAS protocols have been reported to be effective for patients undergoing hepatectomy since they lead to shorter hospitalization duration and reduced morbidity [6-10], although the quality and level of evidence for their efficacy are still lower than those for ERAS protocols for patients undergoing colorectal surgery. Therefore, we performed a randomized controlled trial (RCT) to determine whether an ERAS protocol is more effective compared to conventional postoperative management in patients undergoing open hepatectomy in our department.

\section{METHODS}

\section{Study setting}

The RCT was conducted between August 2014 and March 2017 at Tohoku University Hospital. The trial was approved by the Institutional Review Board of Tohoku University (No. 2014-2-047) and it was registered with the UMIN Clinical Trials Registry (UMIN000039228). All patients signed informed consent to participate in the randomized treatment.

\section{Patients}

All adults who underwent liver resection at our department were eligible. Patients were excluded if they had previously undergone upper digestive surgery, if they required additional procedures (e.g., bile duct reconstruction), if their body mass index was $\leq 18$ or $\geq 30 \mathrm{~kg} / \mathrm{m}^{2}$, if their performance status was $\geq 2$, or if they did not consent to participate. After hospital admission, the study was described in detail to the patients, followed by a comprehensive discussion, before consent was obtained. Patients were randomized into 2 groups by allocation in sealed envelopes.

\section{Perioperative care}

The perioperative care of patients in the ERAS and conventional management groups is summarized in Table 1. Patients in both groups underwent preoperative nutritional evaluation at admission. Patients received additional nutritional supplements if their Controlling Nutritional Status (CONUT) score was $\geq 2$ [11]. Patients in the ERAS group were allowed to consume solid foods until supper the day before surgery and clear water until 2 hours before the induction of anesthesia. Patients in this

Table 1. Perioperative protocols used in the ERAS and conventional management groups

\begin{tabular}{|c|c|c|}
\hline Varaible & ERAS & Conventional management \\
\hline $\begin{array}{l}\text { Preoperative nutritional } \\
\text { care }\end{array}$ & Oral nutritional supplements CONUT score $>2$ & $\begin{array}{l}\text { Oral nutritional supplements CONUT score } \\
>2\end{array}$ \\
\hline Synbiotics & $\begin{array}{l}65 \mathrm{~mL} \text { of probiotic drink and } 3 \text { packs of GFO from } \\
\text { preoperative day } 3 \text { to } 1\end{array}$ & None \\
\hline Diet before surgery & $\begin{array}{l}\text { Normal diet on the day before surgery } \\
250 \mathrm{~mL} \text { of oral carbohydrate solution the night before } \\
\text { surgery and } 2 \mathrm{hr} \text { before anesthesia }\end{array}$ & $\begin{array}{l}\text { Food stopped at lunch the day before } \\
\text { surgery } \\
\text { Clear fluid allowed until } 2 \mathrm{hr} \text { before } \\
\text { anesthesia }\end{array}$ \\
\hline Bowel preparation & $\begin{array}{l}\text { Normal diet on the day before surgery } \\
250 \mathrm{~mL} \text { of oral carbohydrate solution Arginade Water }{ }^{\mathrm{a})} \text { the } \\
\text { night before surgery and } 2 \mathrm{hr} \text { before anesthesia }\end{array}$ & Magnesium citrate the day before surgery \\
\hline During surgery & $\begin{array}{l}\text { Standard anesthetic protocol and surgical management } \\
\text { including hypothermia prevention }\end{array}$ & $\begin{array}{l}\text { Standard anesthetic protocol and surgical } \\
\text { management including hypothermia } \\
\text { prevention }\end{array}$ \\
\hline $\begin{array}{l}\text { Antibiotic prophylaxis } \\
\text { during surgery }\end{array}$ & $\begin{array}{l}\text { Cefmetazole } 1 \mathrm{~g} \text { before skin incision and every } 3 \mathrm{hr} \text { during } \\
\text { surgery }\end{array}$ & $\begin{array}{l}\text { Cefmetazole } 1 \mathrm{~g} \text { before skin incision and } \\
\text { every } 3 \mathrm{hr} \text { during surgery }\end{array}$ \\
\hline Nasogastric tube & Removed before extubating & Removed on POD 1 \\
\hline Postoperative analgesia & $\begin{array}{l}\text { Thoracic epidural analgesia for laparotomy and celecoxib } \\
\text { Acetaminophen for breakthrough pain }\end{array}$ & $\begin{array}{l}\text { Thoracic epidural analgesia for laparotomy } \\
\text { and celecoxib } \\
\text { Acetaminophen for breakthrough pain }\end{array}$ \\
\hline Oral intake & $\begin{array}{l}\text { Water permitted on POD } 1 \\
\text { Normal diet on POD } 2\end{array}$ & $\begin{array}{l}\text { Water permitted after first flatus } \\
\text { Normal diet permitted the next day }\end{array}$ \\
\hline $\begin{array}{l}\text { Postoperative systemic } \\
\text { laxatives }\end{array}$ & Oral magnesium hydroxide from POD 1 & None \\
\hline Postoperative antibiotics & Cefmetazole 2 g per day until POD 2 & Cefmetazole 2 g per day until POD 2 \\
\hline $\begin{array}{l}\text { Postoperative } \\
\text { rehabilitation }\end{array}$ & Physical therapist or bedside nurse & Bedside nurse \\
\hline
\end{tabular}

ERAS, enhanced recovery after surgery; CONUT, Controlling Nutritional Status; POD, postoperative day; GFO, glutamine fiber oligosaccharide.

a) Nestlé Japan Ltd., Kobe, Japan. 
group also received $65 \mathrm{~mL}$ of a commercially available probiotic drink (Yakult, Yakult Honsha, Tokyo, Japan) containing at least $4 \times 10^{10}$ Lactobacillus casei Shirota, 3 packs of glutamine fiber oligosaccharide (GFO, Otsuka Pharmaceutical Factory Inc., Tokushima, Japan), and 3 probiotic tablets (BIO-THREE, Toa Pharmaceutical Co. Ltd., Takasaki, Japan) containing 3 different kinds of useful bacteria on preoperative days 3, 2, and 1. Additionally, patients in the ERAS group underwent carbohydrate loading with 2 packs of 125-mL Arginade Water (Nestlé Japan Ltd., Kobe, Japan) during the evening before surgery.

Patients in the conventional management group could take in clear fluids until the evening and solid foods until lunchtime the day before surgery. Oral mechanical bowel preparation with magnesium citrate was performed during the afternoon of the day before surgery. Patients in both groups did not receive preoperative anesthetic premedication.

\section{Intraoperative care}

Patients in both groups underwent the same anesthetic procedures. Cefmetazole ( $1 \mathrm{~g}$ ) was administered as a routine antibiotic prophylaxis every 3 hours during the surgery. A thoracic epidural was used in patients who satisfied the institutional guidelines. Normothermia was maintained with a forced-air warming blanket. Intermittent pneumatic leg compression devices were used in all patients.

\section{Surgical technique}

All patients underwent surgery performed by 1 of 2 consultant surgeons in our department. The attending surgeon decided whether to make a J-shaped subcostal incision or a Bentz incision. All surgical procedures were performed using similar techniques. The liver parenchyma was transected by ultrasonic dissection with a Cavitron Ultrasonic Surgical Aspirator (Valleylab, Boulder, CO, USA). The exact procedure and the need for the Pringle maneuver were determined by the surgeon. Before performing the Pringle maneuver, hydrocortisone $(100 \mathrm{mg}$ ) was administered intravenously to alleviate ischemia-reperfusion injury [12]. An abdominal pleated drain was inserted in all patients.

\section{Postoperative care}

Immediately after surgery, all patients were extubated and transferred to the intensive care unit. For the ERAS group, the nasogastric tube was removed immediately after extubating, while for the conventional management group, it was removed on postoperative day (POD) 1. Patients in both groups received fluid optimization according to conventional markers of hypovolemia, including pulse rate, central venous pressure, urine output, and arterial lactate levels. Maintenance fluids were started at $1-2 \mathrm{~mL} / \mathrm{kg} /$ hour. Magnesium oxide was routinely administered as a postoperative laxative in the ERAS group. In terms of postoperative rehabilitation, patients in the ERAS group were mobilized by either the physical therapist or the bedside nurse. Patients in the conventional group were mobilized by the bedside nurse.

\section{Primary outcome}

The primary outcome was the amount of time that elapsed before patients were considered medically fit for discharge (MFD). MFD was defined as follows; sufficient postoperative pain control with oral analgesia, tolerance of solid food, free from fluid infusion, passage of feces, and recovery to preoperative performance status.

\section{Secondary outcome}

Secondary outcomes were the incidences of postoperative complications, mortality, insulin resistance, nutritional status, time to passage of first flatus and defecation, time to mobilization, time to freedom from infusion, and postoperative energy status determined by indirect calorimetry. Complications were graded according to the Clavien-Dindo classification [13]. Mortality was defined as death occurring in the hospital or within 30 days after surgery. Insulin resistance was assessed with the homeostasis model assessment of insulin resistance (HOMA-IR) using the following formula: HOMA-IR = fasting plasma insulin level $(\mathrm{pU} / \mathrm{mL}) \times$ fasting plasma glucose $(\mathrm{mg} /$ $\mathrm{dL} / 22.5$ [14]. The nutritional status of patients was assessed using the CONUT score [11]. Serum albumin, prealbumin, transferrin, branched amino acids/tyrosine molecular ratio, and CRP were measured before surgery and on POD 1, 3, 5 , and 7. The times to first flatus, bowel opening, mobilization, and freedom from infusion were recorded. Patients underwent indirect calorimetry preoperatively, and on POD 7, the resting energy expenditure (REE) [15] and respiratory quotient (RQ) [16] were determined.

\section{Statistical analysis}

The sample size calculation was based on the difference in postoperative LOHS determined in a retrospective audit of a pilot study at our department. Before ERAS induction, the MFD of most patients who underwent hepatectomy was approximately 15 days. It was assumed that a clinically significant reduction in MFD would be 1 day. The sample size was calculated with a power of $80 \%$ using a 2-sided 2-sample Student t-test. The results of our calculation indicated that a minimum of 36 patients were required (18 per group). Continuous data with a normal distribution were compared between the 2 groups using a 2-sample Student t-test. Data with skewed distributions were analyzed using the MannWhitney U-test. Fisher exact test or chi-square test was used for categorical variables. Statistical analyses were performed using 
JMP for Mac ver. 13 (SAS Institute, Cary, NC, USA). A P-value of $<0.05$ was considered statistically significant.

\section{RESULTS}

Altogether, 111 consecutive patients were referred for hepatectomy at our department during the study period. Ten patients were excluded because they did not provide informed consent. Additionally, 12 patients who were scheduled to undergo hepatectomy with biliary reconstruction, 1 patient with glycogen storage disease who needed special perioperative care, 1 patient who underwent gastrectomy, 2 patients who underwent emergent hepatectomy, 3 patients who underwent simultaneous colon or rectal surgery, and 24 patients who underwent laparoscopic hepatectomy were excluded from the study. Therefore, 57 patients were enrolled in this study. Twenty-nine of these patients were randomly allocated to the ERAS group and the remaining 28 were allocated to the conventional management group (Fig. 1).

Patient characteristics are summarized in Table 2. Both groups were similar with respect to age, sex, body mass index, Child-Pugh classification, indocyanine green retention at 15 minutes, viral status, disease, operating time, duration of Pringle maneuver, and blood loss. Hepatectomy segment $\geq$

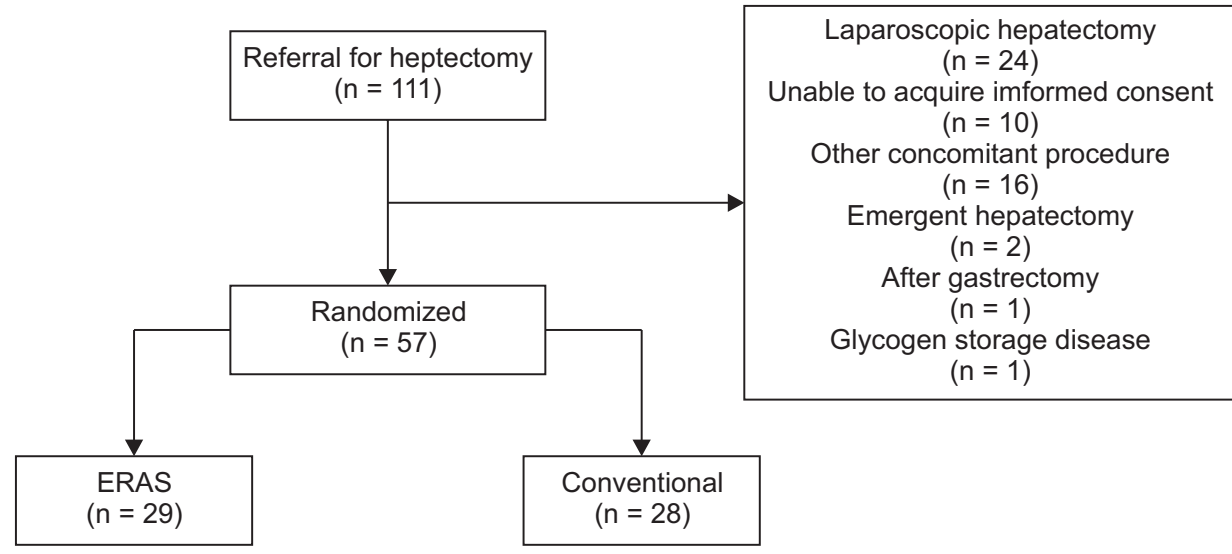

Fig. 1. Patient disposition. ERAS, enhanced recovery after surgery.

Table 2. Baseline characteristics and surgical details

\begin{tabular}{|c|c|c|c|}
\hline Varable & ERAS group $(n=29)$ & Conventional management group $(n=28)$ & P-value \\
\hline Age $(y r)$ & $68(51-75)$ & $67(60-75)$ & 0.433 \\
\hline Sex (male/female) & $17 / 12$ & $20 / 8$ & 0.309 \\
\hline Body mass index $\left(\mathrm{kg} / \mathrm{m}^{2}\right)$ & $23.2(19.4-25.5)$ & $24.0(20.4-25.5)$ & 0.690 \\
\hline Child-Pugh classification (A/B) & $29 / 0$ & $28 / 1$ & 0.230 \\
\hline ICG R15 (\%) & $11.0(7.6-18.4)$ & $9.9(6.4-18.7)$ & 0.489 \\
\hline Virus (B/C/nonB-nonC) & $9 / 5 / 15$ & $7 / 5 / 16$ & 0.875 \\
\hline \multicolumn{4}{|l|}{ Disease } \\
\hline $\mathrm{HCC}$ & $22(75.9)$ & $18(64.3)$ & \\
\hline Colorectal metastases & $7(24.1)$ & $7(25.0)$ & \\
\hline Benign disease & $0(0)$ & $1(3.6)$ & \\
\hline \multicolumn{4}{|l|}{ Surgical details } \\
\hline \multicolumn{4}{|l|}{ Range of hepatectomy } \\
\hline \multicolumn{4}{|l|}{ Hepatectomy segments } \\
\hline$\geq 2$ & $3(10.3)$ & $9(32.1)$ & 0.040 \\
\hline$<2$ & $26(89.7)$ & $19(67.9)$ & \\
\hline Operating time (min) & $324(274-385)$ & $349(283-448)$ & 0.091 \\
\hline Blood loss $(\mathrm{mL})$ & $588(336-1,188)$ & $748(377-1,781)$ & 0.101 \\
\hline Duration of Pringle maneuver (min) & $40(25-73)$ & $51(35-75)$ & 0.246 \\
\hline Postoperative complication ${ }^{\text {a) }}$ & $1(3.4)$ & $4(14.2)$ & 0.136 \\
\hline Death & $0(0)$ & $0(0)$ & \\
\hline
\end{tabular}

Values are reported as median (range), number only, or number (\%).

ERAS, enhanced recovery after surgery; ICG R15, indocyanine green retention at 15 minutes; HCC, hepatocellular carcinoma.

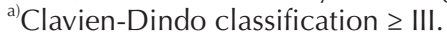


2 was performed in a greater proportion of patients in the conventional management group than in the ERAS group ( $32 \%$ vs. $10 \%$, respectively; $\mathrm{P}=0.040$ ).

The median time to MFD, the primary endpoint, was not significantly reduced in the ERAS group compared to the conventional management group (6.5 vs. 7 days, respectively; $P$ $=0.382$ ). Furthermore, the median LOHS was not significantly shorter in the ERAS group (13 vs. 13 days, respectively; $\mathrm{P}=$ 0.373).
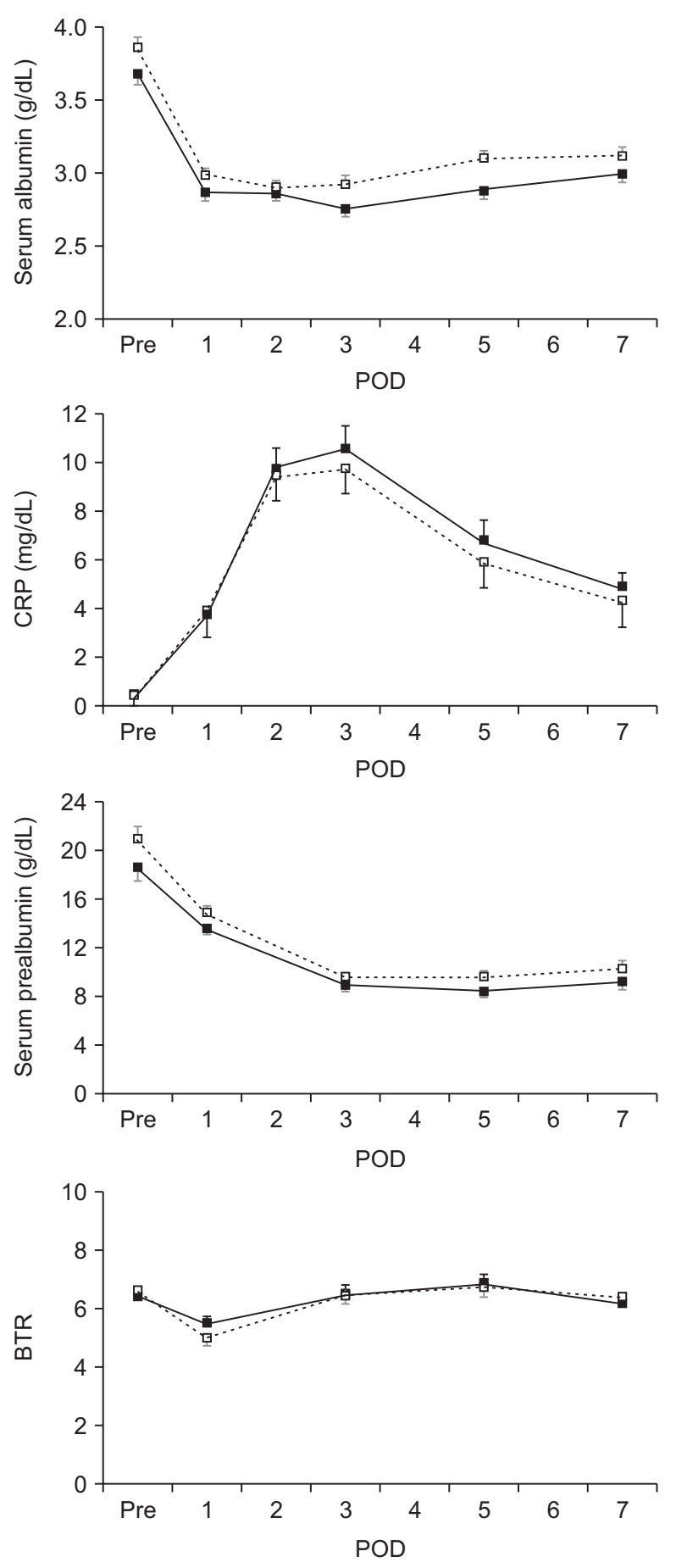

There were no deaths in either group. The proportion of patients with complications scoring $\geq 3$ on the Clavien-Dindo classification was not significantly different between the ERAS and conventional management groups (3.4\% vs. $14.2 \%$, respectively; $\mathrm{P}=0.136)$. The times to first flatus $(1.8 \mathrm{vs} .2 .4$ days, respectively; $\mathrm{P}=0.004$ ) and first defecation (2.8 vs. 3.7 days, respectively; $P=0.021$ ) were significantly shorter in the ERAS group. However, the median times to first standing ( 2 vs. 2 days, respectively; $P=0.343$ ), first walk ( 2 vs. 2 days,
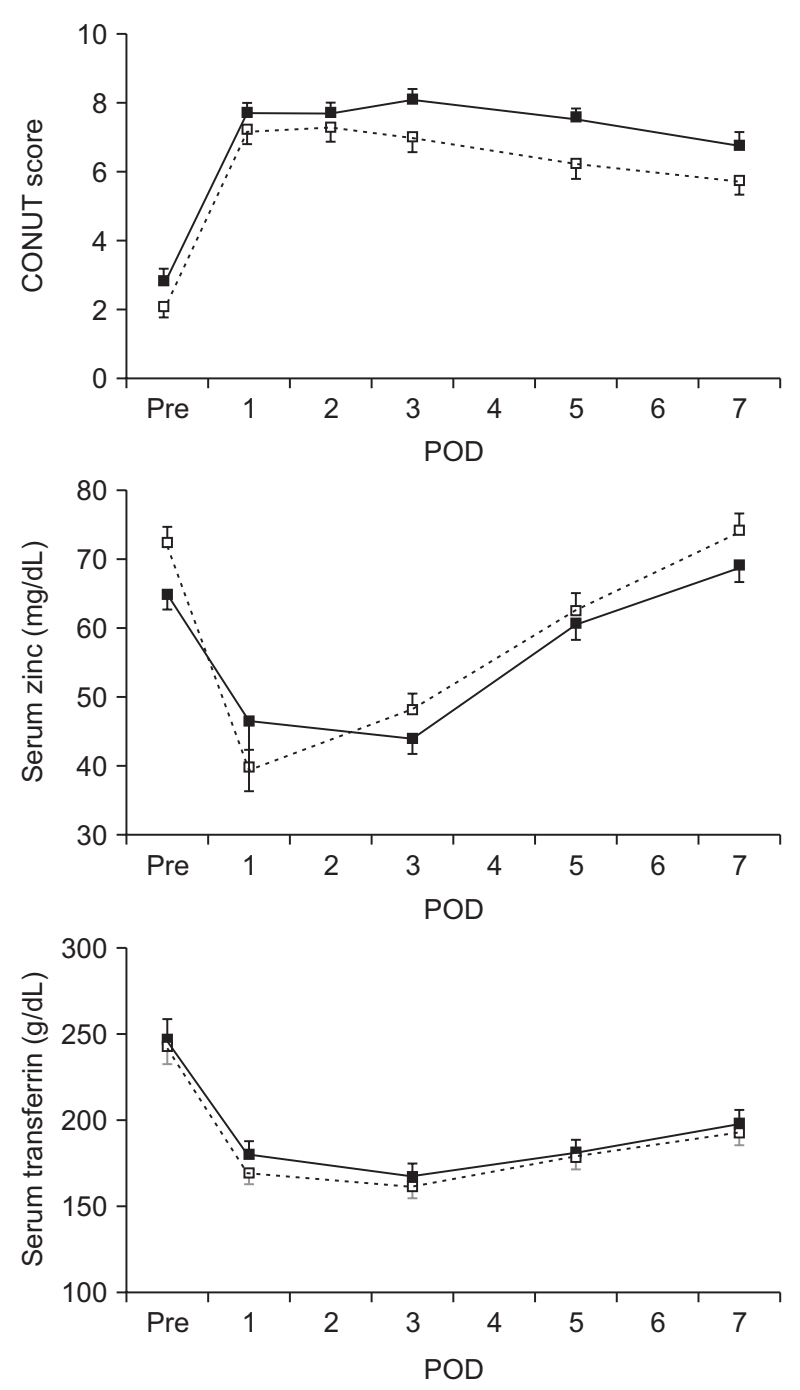

- ERAS group

Conventional group 
respectively; $\mathrm{P}=0.991$ ), and freedom from infusion ( 5 vs. 5.5 days, respectively; $\mathrm{P}=0.162$ ) were not significantly different between the ERAS and conventional management groups.

There were no differences in serum albumin, prealbumin, transferrin, branched amino acids/tyrosine molecular ratio, and CRP at any point (Fig. 2). Insulin resistance, in terms of HOMAIR, is shown in Fig. 3. There were no significant differences in HOMA-IR between the 2 groups at any time point, although there was a trend towards a greater improvement in insulin resistance on POD 1 in the ERAS group (5.5 vs. 11.0, respectively; $\mathrm{P}=0.055)$.

The RQ decreased significantly during the postoperative period (Fig. 4A), although it was not significantly different between the 2 groups (Fig. 4B). The REE was not significantly different before surgery and on POD 7 in all patients combined (Fig. 4C) and between the 2 groups (Fig. 4D).

\section{DISCUSSION}

We first implemented an ERAS protocol for patients

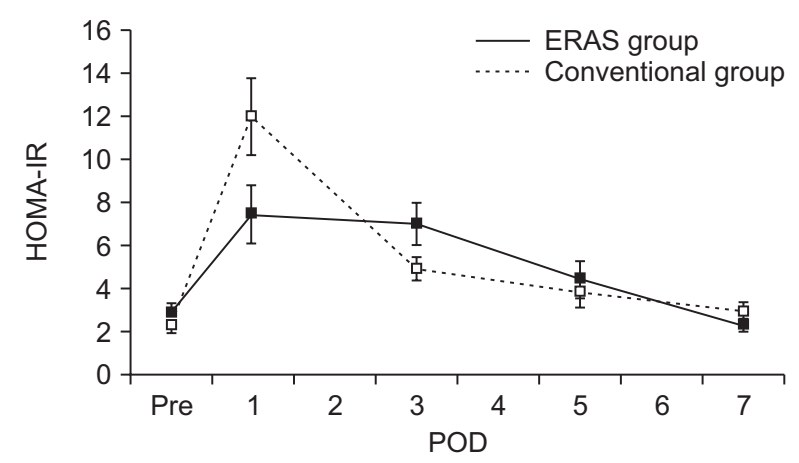

Fig. 3 Mean (standard error of the mean) changes in homeostasis model assessment of insulin resistance (HOMAIR) from before surgery to postoperative day (POD) 7. Pre, preoperative. undergoing open hepatectomy in 2013. The ERAS protocol is a multidisciplinary approach, and medical staff must understand the efficacy of the ERAS protocol to implement it correctly. In this study, we sought to confirm the efficacy of our ERAS protocol in comparison to that of conventional management in an RCT. As for the primary endpoint of the study, the MFD and LOHS were similar in the ERAS and conventional groups, although previous studies reported that enhanced care protocols helped reduce these outcomes in patients undergoing open hepatectomy $[17,18]$. In the present study, bowel preparation, duration of nasogastric tube, postoperative oral intake, and laxatives, which seemed to be associated with recovery from postoperative paresis, differed between the 2 groups. A reason for this finding could be that these differences between the 2 groups might not have been significant enough to reduce MFD. Another reason is that the quality of the postoperative mobilization was similar in both groups. In the ERAS group, the physical therapist intervened before surgery; however, they could intervene for only 2 days. Moreover, the bedside nurse comparably mobilized the patients in the conventional group after surgery.

This study suggested that the ERAS protocol might be effective in improving recovery from postoperative paresis and postoperative insulin resistance, although the differences in these outcomes did not reach statistical significance. These findings suggest that some components of conventional management, such as mechanical bowel preparation and keeping the nasogastric tube in place for several days after surgery, are unnecessary in patients undergoing open hepatectomy. Routine nasogastric tube intubation is not recommended because it is not beneficial and may cause pulmonary complications in patients undergoing elective hepatectomy [19]. This study revealed that keeping the nasogastric tube in place after surgery has no positive effects. Therefore, it should be removed after surgery.

Postoperative laxatives and early food intake in the ERAS
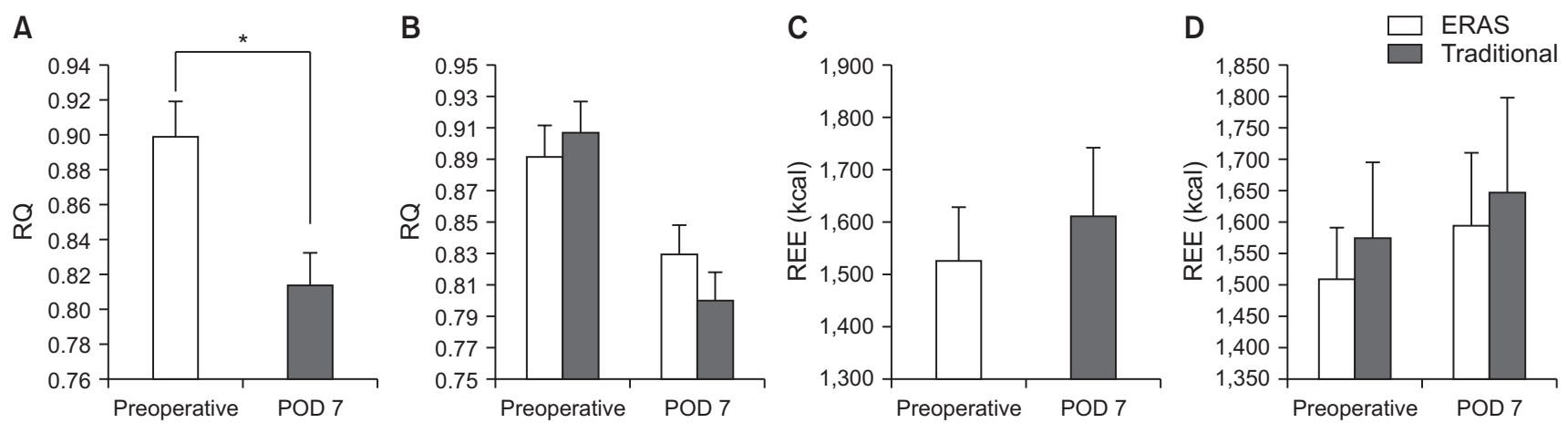

Fig. 4 Indirect calorimetry. (A) Comparison of respiratory quotient (RQ) before surgery and on postoperative day (POD) 7 in all patients. (B) Comparison of RQ between the enhanced recovery after surgery (ERAS) and conventional management groups before surgery and on POD 7. (C) Comparison of resting energy expenditure (REE) before surgery and on POD 7 in all patients. (D) Comparison of REE between the ERAS and conventional management groups before surgery and on POD 7. ${ }^{* P}<0.001$. 
Table 3. Postoperative outcomes

\begin{tabular}{lccc}
\hline \multicolumn{1}{c}{ Time to (day) } & ERAS group $(\mathrm{n}=29)$ & Conventional management group $(\mathrm{n}=28)$ & P-value \\
MFD & $6.5(5-7)$ & $7(6-9)$ & 0.382 \\
LOHS & $13(10-15)$ & $13(11-16)$ & 0.373 \\
First flatus & $1.8 \pm 0.13$ & $2.4 \pm 0.14$ & 0.004 \\
First defecation & $2.8 \pm 0.22$ & $3.7 \pm 0.23$ & 0.021 \\
First standing & $2(2-3)$ & $2(2-3)$ & 0.343 \\
First walking & $2(2-3)$ & $2(2-3)$ & 0.991 \\
Freedom from infusion & $5(4.25-6)$ & $5.5(5-8)$ & 0.162 \\
\hline
\end{tabular}

Values are reported as median (range) or mean \pm standard error.

ERAS, enhanced recovery after surgery; MFD, medically fit for discharge; LOHS, length of hospital stay.

group seemed to contribute to earlier passage of flatus and stool without any adverse events, although these did not reduce the MFD and LOHS (Table 3). These results are consistent with those of a previous report [20]. Although stimulation of bowel movement with postoperative laxatives is not recommended in the ERAS guidelines [6], it may be an important component of ERAS protocols for the shortening of the postoperative ileus period. Oral hydroxy magnesium is a low-cost drug, and may be considered part of the ERAS protocol for hepatectomy.

Preoperative mechanical bowel preparation with magnesium citrate was routinely performed for patients undergoing hepatectomy at our department for many years. Bowel preparation might reduce bowel content and facilitate surgical procedures. However, we found no differences in bleeding volume or operation time between the 2 groups in this study. Furthermore, the surgeons did not observe any differences between the 2 groups during the operations. Thus, bowel preparation is unnecessary in patients undergoing hepatectomy.

Although synbiotics have been reported to reduce postoperative infectious complications and are recommended for surgical patients [21-24], they did not have a positive effect on postoperative complications in our study. In this study, synbiotics were administered for 3 days before surgery; a longer period of administration may be needed to confer beneficial effects.

Preoperative carbohydrate loading was reported to attenuate postoperative insulin resistance [25]. In this study, we found that carbohydrate loading seemed to have a positive effect on insulin resistance on POD 1, corresponding to the peak insulin resistance time. Considering that carbohydrate loading may assist with recovery after gastrointestinal paresis, preoperative carbohydrate loading should be recommended for hepatectomy.

Metabolic changes occur after major surgery, and indirect calorimetry can detect changes in REE and RQ. A low postoperative RQ indicates a hypercatabolic state, which could lead to sarcopenia. Although the ERAS protocol used in this study did not affect the postoperative metabolic state, nutritional support should be considered after hepatectomy because sarcopenia is associated with poor outcomes [26,27].

The present study had several limitations. First, the sample size was relatively small. Second, despite randomization, there were some baseline differences between the 2 groups (Table 2). In particular, the range of hepatectomy was greater in the conventional group $(P=0.040)$. This difference could cause worse postoperative liver function and delayed postoperative recovery from paresis in the conventional group, although in both groups, the operative time and blood loss were not significantly different. Third, the present RCT was not performed in a double-blind manner because of the nature of the intervention. Moreover, the medical staff involved in this study also experienced difficulty with its implementation because they were familiar with the ERAS protocol and felt that conventional care is suboptimal.

In conclusion, this RCT did not demonstrate greater efficacy of the ERAS protocol in reducing MFD and LOHS in patients undergoing open hepatectomy. However, the results suggest that the ERAS protocol may confer benefits in terms of accelerating postoperative recovery from gastrointestinal paresis.

\section{ACKNOWLEDGEMENTS}

\section{Conflict of Interest}

No potential conflict of interest relevant to this article was reported.

\section{ORCID iD}

Wataru Nakanishi: https://orcid.org/0000-0002-7034-9764

Shigehito Miyagi: https://orcid.org/0000-0002-7628-3487

Kazuaki Tokodai: https://orcid.org/0000-0002-0607-8250

Atsushi Fujio: https://orcid.org/0000-0002-2198-7508

Kengo Sasaki: https://orcid.org/0000-0002-3244-2845

Yoshihiro Shono: https://orcid.org/0000-0002-0804-285X

Michiaki Unno: https://orcid.org/0000-0002-2145-6416

Takashi Kamei: https://orcid.org/0000-0003-1282-0463 
Author Contribution

Conceptualization: WN, SM, KT

Formal Analysis: WN, KT

Investigation: WN, AF, KS, YS
Methodology: SM, KT, MU, TK

Project Administration: SM

Writing - Original Draft: WN

Writing - Review \& Editing: SM, KT, AF, KS, YS, MU, TK

\section{REFERENCES}

1. Lassen K, Coolsen MM, Slim K, Carli F, de Aguilar-Nascimento JE, Schäfer $\mathrm{M}$, et al. Guidelines for perioperative care for pancreaticoduodenectomy: Enhanced Recovery After Surgery (ERAS®) Society recommendations. Clin Nutr 2012;31:81730.

2. Martin D, Roulin D, Grass F, Addor V, Ljungqvist $\mathrm{O}$, Demartines $\mathrm{N}$, et al. A multicentre qualitative study assessing implementation of an Enhanced Recovery After Surgery program. Clin Nutr 2018;37: 2172-7.

3. Lv L, Shao YF, Zhou YB. The enhanced recovery after surgery (ERAS) pathway for patients undergoing colorectal surgery: an update of meta-analysis of randomized controlled trials. Int J Colorectal Dis 2012;27:1549-54.

4. Ota H, Ikenaga M, Hasegawa J, Murata K, Miyake Y, Mizushima T, et al. Safety and efficacy of an "enhanced recovery after surgery" protocol for patients undergoing colon cancer surgery: a multi-institutional controlled study. Surg Today 2017;47:66875.

5. Wei AC, Greig PD, Grant D, Taylor B, Langer B, Gallinger S. Survival after hepatic resection for colorectal metastases: a 10-year experience. Ann Surg Oncol 2006;13:668-76.

6. Melloul E, Hübner M, Scott M, Snowden C, Prentis J, Dejong $\mathrm{CH}$, et al. Guidelines for perioperative care for liver surgery: Enhanced Recovery After Surgery (ERAS) Society recommendations. World J Surg 2016:40:2425-40.

7. Sánchez-Pérez B, Aranda-Narváez JM, Suárez-Muñoz MA, Eladel-Delfresno M, Fernández-Aguilar JL, Pérez-Daga JA, et al. Fast-track program in laparoscopic liver surgery: theory or fact? World J Gastrointest Surg 2012;4:246-50.

8. Dunne DF, Yip VS, Jones RP, McChesney EA, Lythgoe DT, Psarelli EE, et al. Enhanced recovery in the resection of colorectal liver metastases. J Surg Oncol 2014;110:197-202.

9. Kaibori M, Matsui K, Ishizaki M, Iida H, Yoshii K, Asano H, et al. Effects of implementing an "enhanced recovery after surgery" program on patients undergoing resection of hepatocellular carcinoma. Surg Today 2017;47:42-51.

10. Fujio A, Miyagi S, Tokodai K, Nakanishi W, Nishimura R, Mitsui K, et al. Effects of a new perioperative enhanced recovery after surgery protocol in hepatectomy for hepatocellular carcinoma. Surg Today 2020;50:615-22.

11. Ignacio de Ulíbarri J, González-Madroño A, de Villar NG, González P, González B, Mancha A, et al. CONUT: a tool for controlling nutritional status. First validation in a hospital population. Nutr Hosp 2005:20:38-45.

12. Hayashi Y, Takayama T, Yamazaki S, Moriguchi M, Ohkubo T, Nakayama H, et al. Validation of perioperative steroids administration in liver resection: a randomized controlled trial. Ann Surg 2011:253:50-5.

13. Dindo D, Demartines N, Clavien PA. Classification of surgical complications: a new proposal with evaluation in a cohort of 6336 patients and results of a survey. Ann Surg 2004:240:205-13.

14. Matthews DR, Rudenski AS, Burnett MA, Darling P, Turner RC. The half-life of endogenous insulin and C-peptide in man assessed by somatostatin suppression. Clin Endocrinol (Oxf) 1985:23:71-9.
15. Dunn DC, Harrison AL, Curtice C, DeLand S, Donnelly B, Fujioka E, et al. The importance of migratory connectivity for global ocean policy. Proc Biol Sci 2019;286:20191472.

16. Hausel J, Nygren J, Lagerkranser M, Hellström PM, Hammarqvist F, Almström $C$, et al. A carbohydrate-rich drink reduces preoperative discomfort in elective surgery patients. Anesth Analg 2001;93:1344-50.

17. Jones C, Kelliher L, Dickinson M, Riga A, Worthington T, Scott MJ, et al. Randomized clinical trial on enhanced recovery versus standard care following open liver resection. Br J Surg 2013;100: 1015-24.

18. van Dam RM, Hendry PO, Coolsen MM, Bemelmans $\mathrm{MH}$, Lassen $\mathrm{K}$, Revhaug A, et al. Initial experience with a multimodal enhanced recovery programme in patients undergoing liver resection. $\mathrm{Br} \mathrm{J}$ Surg 2008:95:969-75.

19. Pessaux P, Regimbeau JM, Dondéro F, Plasse M, Mantz J, Belghiti J. Randomized clinical trial evaluating the need for routine nasogastric decompression after elective hepatic resection. Br J Surg 2007:94:297-303.

20. Hendry PO, van Dam RM, Bukkems SF, McKeown DW, Parks RW, Preston T, et al. Randomized clinical trial of laxatives and oral nutritional supplements within an enhanced recovery after surgery protocol following liver resection. Br J Surg 2010;97:1198-206.

21. Sugawara G, Nagino M, Nishio H, Ebata T, Takagi K, Asahara T, et al. Perioperative synbiotic treatment to prevent postoperative infectious complications in biliary cancer surgery: a randomized controlled 
trial. Ann Surg 2006;244:706-14.

22. Rayes N, Pilarski T, Stockmann M, Bengmark S, Neuhaus P, Seehofer D. Effect of pre- and probiotics on liver regeneration after resection: a randomised, double-blind pilot study. Benef Microbes 2012;3:237-44.

23. Usami M, Miyoshi M, Kanbara Y, Aoyama M, Sakaki H, Shuno K, et al. Effects of perioperative synbiotic treatment on infectious complications, intestinal integrity, and fecal flora and organic acids in hepatic surgery with or without cirrhosis. JPEN J Parenter Enteral Nutr
2011;35:317-28.

24. Wu XD, Liu MM, Liang X, Hu N, Huang W. Effects of perioperative supplementation with pro-/synbiotics on clinical outcomes in surgical patients: a meta-analysis with trial sequential analysis of randomized controlled trials. Clin Nutr 2018;37:505-15.

25. Soop M, Nygren J, Myrenfors P, Thorell A, Ljungqvist $O$. Preoperative oral carbohydrate treatment attenuates immediate postoperative insulin resistance. Am J Physiol Endocrinol Metab 2001;280: E576-83.

26. Kobayashi A, Kaido T, Hamaguchi
Y, Okumura S, Taura K, Hatano E, et al. Impact of postoperative changes in sarcopenic factors on outcomes after hepatectomy for hepatocellular carcinoma. J Hepatobiliary Pancreat Sci 2016;23:57-64.

27. Okumura S, Kaido T, Hamaguchi Y, Fujimoto Y, Kobayashi A, Iida T, et al. Impact of the preoperative quantity and quality of skeletal muscle on outcomes after resection of extrahepatic biliary malignancies. Surgery 2016;159:821-33. 